\title{
Simpler and Faster Development of Tumor
}

\section{Phylogeny Pipelines}

\author{
Sarwan Ali, ${ }^{1 \dagger}$ Simone Ciccolella, ${ }^{2 \dagger}$ \\ Lorenzo Lucarella, ${ }^{2}$ Gianluca Della Vedova, ${ }^{2+}$ Murray Patterson ${ }^{1+*}$ \\ ${ }^{1}$ Department of Computer Science, Georgia State University, \\ Atlanta, GA 30303, USA \\ ${ }^{2}$ Department of Informatics, Systems, and Communications, \\ University of Milano - Bicocca \\ viale Sarca 336, Milano, Italy \\ $\dagger$ Joint first authors; + Joint last authors \\ *Corresponding author \\ email: sali85@student.gsu.edu; simone.ciccolella@unimib.it; \\ l.lucarella@campus.unimib.it; gianluca.dellavedova@unimib.it; \\ mpatterson30@gsu.edu
}

August 30, 2021

Keywords: Single Cell Sequencing, Cancer Analysis, Autoencoder, Ridge Regression, Dimensionality Reduction

\begin{abstract}
In the recent years there has been an increasing amount of single-cell sequencing (SCS) studies, producing a considerable
\end{abstract}


number of new datasets. This has particularly affected the field of cancer analysis, where more and more papers are published using this sequencing technique that allows for capturing more detailed information regarding the specific genetic mutations on each individually sampled cell.

As the amount of information increases, it is necessary to have more sophisticated and rapid tools for analyzing the samples. To this goal we developed plastic, an easy-to-use and quick to adapt pipeline that integrates three different steps: (1) to simplify the input data; (2) to infer tumor phylogenies; and (3) to compare the phylogenies. We have created a pipeline submodule for each of those steps, and developed new in-memory data structures that allow for easy and transparent sharing of the information across the tools implementing the above steps.

While we use existing open source tools for those steps, we have extended the tool used for simplifying the input data, incorporating two machine learning procedures — which greatly reduce the running time without affecting the quality of the downstream analysis. Moreover, we have introduced the capability of producing some plots to quickly visualize results.

\section{Introduction}

During the last few years we have witnessed an explosion of computational tools to infer tumor phylogenies (also called cancer progressions) from singlecell sequencing (SCS) data.

Most of the algorithmic research has so far focused on bulk sequencing data 
for inferring tumor phylogenies, mainly because of the widespread availability and affordability of the next-generation sequencing (NGS) data that are used, producing a large number of tools Strino et al. (2013); Jiao et al. (2014); Hajirasouliha et al. (2014); Yuan et al. (2015); Popic et al. (2015); Malikic et al. (2015); El-Kebir et al. (2016); Marass et al. (2016); Satas and Raphael (2017); Bonizzoni et al. (2018); Toosi et al. (2019); Wu (2019). From a computational point of view, the main characteristic of bulk sequencing data is that only the approximate proportion of cells with any given mutation is observable, without distinguishing the cells that carry them. Moreover, each sample contains a mixture of both healthy and cancerous cells — the latter belonging to different and unknown clones — therefore further introducing uncertainty.

More recently, the introduction of single-cell sequencing (SCS) technologies promises to greatly reduce such uncertainty, since the presence or absence of mutation is determined at the level of the cell. Unfortunately, SCS is still much more expensive than bulk sequencing, hence limiting its adoption in practice. Moreover, the quality of the data obtained from SCS is not yet at par with bulk sequencing Kharchenko (2021). In fact, those datasets are affected by some clearly identified problems: (1) doublet cell captures, that is, data originating from two cells instead of one; (2) false negatives from allelic dropout, that is, the presence of a mutation is not detected; and (3) missing values due to low coverage. However, all three of these problems are slowly fading away — the latter two driven by the reduction in cost that allows a higher coverage, and the first due to the development of state-of-the art approaches DePasquale et al. (2019) are able to remove such artifacts.

Various methods have been recently developed for inferring tumor phylogenies given current SCS data Jahn et al. (2016); Ross and Markowetz (2016); Zafar et al. 2017, 2019); Ciccolella et al. (2020b); El-Kebir (2018); Singer et al. 
(2018), some of them introducing a hybrid approach of combining both SCS and VAF (variant allele frequency, from bulk sequencing) data Ramazzotti et al. (2019); Malikic et al. (2017); Salehi et al. (2017). To trim the search space and reduce the time needed to infer a phylogeny, most methods rely on the infinite sites assumption (ISA), which essentially states that each mutation is acquired at most once in the phylogeny and is never lost. This assumption leads to a computationally tractable model of evolution called perfect the phylogeny Gusfield (1991); Kimura (1969).

However, some studies Kuipers et al. (2017); Brown et al. (2017) on cancer data provide strong hints that the ISA does not always hold, the main reason is that cancers usually have large independent deletions on separate branches of the phylogeny Brown et al. (2017). When those deletions span a shared locus, we observe multiple deletions of the same mutation.

Relaxing the ISA greatly expands the search space, making it more difficult to develop efficient approaches. For this reason, the number of possible mutation losses is usually bounded in any (more general) model which relaxes the ISA. While the general Dollo model Farris (1977); Rogozin et al. (2006) does not impose any restriction on the number of losses, more restricted models are the Dollo- $k$ and the Dollo-1 (also known as the persistent phylogenyBonizzoni et al. (2012, 2017)).

Even though relaxing the ISA increases the complexity, some methods have appeared, such as TRaIT Ramazzotti et al. (2019), SiFit Zafar et al. (2017), SASC Ciccolella et al. (2020b) and SPhyR El-Kebir 2018). The latter is extremely relevant to our context, as it introduces the idea of clustering the input SCS matrix to reduce the running time. In Ciccolella et al. (2021) this idea is further developed, by devising a clustering method tailored to SCS data - while SPhyR instead relied on the ubiquitous $k$-means McQueen (1967); Anderberg 
(1973) algorithm. Since SCS data are becoming cheaper to produce, we expect the datasets to increase more rapidly than computing power, as mentioned explicitly in Kharchenko (2021). Therefore including some steps to reduce the instance matrix will become even more common in the next years.

The availability of so many tools for inferring tumor phylogenies, not to mention the parameters that those methods routinely have, means that it is easy to have several different phylogenies from the same SCS dataset, motivating the search for methods that are able to compare and cluster those phylogenies - a large cluster with several highly similar phylogenies is likely to be more reliable, since the underlying evolution is confirmed by multiple methods. In this direction, some methods to measure the distance between two tumor phylogenies have recently been proposed DiNardo et al. (2019); Karpov et al. (2019); Govek et al. (2018); Bernardini et al. (2019, 2020); Ciccolella et al. (2020a); Jahn et al. (2021). While these measures vary greatly in practical applicability, they all express the need for incorporating the evolutionary process into the definition of distance.

Our discussion so far shows that tumor phylogeny inference is crystallizing into different, well-established steps that are combined to obtain a complete tool that starts from an SCS dataset and ends with one or more phylogenies together with some rough idea of their relationships. Still, how to combine those steps is largely ad-hoc, making it more time consuming than is necessary to develop a complete pipeline.

With the goal of making the analysis of cancer data more streamlined, we developed plastic (PipeLine Amalgamating Single-cell Tree Inference Components), an integrated and easy to use tool that includes clustering, phylogeny inference, and comparison steps. The plastic tool is developed in Python and can be easily integrated into any script or used inside an interactive notebook, 
such as Jupyter Notebook, to facilitate the reproduction of research results. Currently, plastic incorporates the publicly available tools celluloid, $S A S C$, and MP3treesim - respectively for the clustering, inference, and distance steps — but provides the infrastructure to easily extend it to incorporate any other tool. In fact, plastic provides unified in-memory data structures to manage the communication between steps.

Moreover, the current strategies for trimming SCS datasets focus on reducing the number of mutations, while leaving the set of cells unchanged. We have divided the clustering step into two parts: first reducing the number of cells, then reducing the number of mutations. We have explored two strategies for the first part that has been proven to be useful in the past, namely, ridge regression Hoerl and Kennard (1970); Marquardt and Snee (1975) and autoencoder Wang et al. (2014).

Ridge regression is a variant of least squares regression, which deals with the trade-off between bias and variance while fitting the regression line. As compared to least squares regression, ridge regression consist of an $l_{2}$ penalty on the regression coefficients (see Equation 1). This penalty term in ridge regression helps to introduce some bias, which eventually helps to reduce the variance while fitting the regression line (hence regularizing the fit). In least squares regression, since we do not have the bias, there will be a high variance while fitting the line. Ridge regression has been successfully used in the literature for dimensionality reduction Chen et al. (2018); Imakura et al. (2019); Liu et al. (2019).

Autoencoder is an unsupervised approach based on artificial neural networks. It takes as input the data matrix, encodes it into a latent space (of reduced dimension), and then tries to reconstruct the original input from the latent space. During the reconstruction process, it tries to minimize least squared error. Autoencoder has been successfully used in the literature for dimensionality reduc- 
tion Ramamurthy et al. (2020); Hu and Greene (2018); D'Agostino et al. (2018); Abeßer et al. (2017). For further detail about ridge regression and autoencoder, please refer to Section 2.1. The idea with both ridge regression and autoencoder is that we can perform downstream clustering (tree inference, etc.) in reduced dimensional data, rather than applying clustering, etc., on the high dimensional data, resulting in reduced downstream runtimes.

We have run two different experiments: one on real data to showcase all features of plastic, including its capability to plot clustering of mutations (i.e., the output of the clustering step) and trees; and the second on simulated data, to assess the reduction in running time stemming from the reduction in the number of cells provided by the two dimensionality reduction strategies mentioned above. The plastic tool and all data needed to reproduce the analysis can be found at https://github.com/plastic-phy, including the source code of the Jupyter notebook used for the real data experiment, witnessing the simplicity of our approach. The plastic tool is available under the MIT license.

\section{Methods}

We have developed an integrated, modular, and extendable tool for inferring and comparing cancer progressions (also referred to as tumor phylogenies) called plastic, which integrates three separate steps into a single program that shares the same data structure among these steps. These three steps are (1) input matrix reduction, (2) tumor phylogeny inference, and (3) tumor phylogeny comparison.

One of the main contributions of our paper is the integration of different tools that usually have specific on-disk input and output file formats, therefore needing a parsing step to process the input, and a dedicated procedure to produce the output. Instead, plastic uses in-memory data structures to share all 


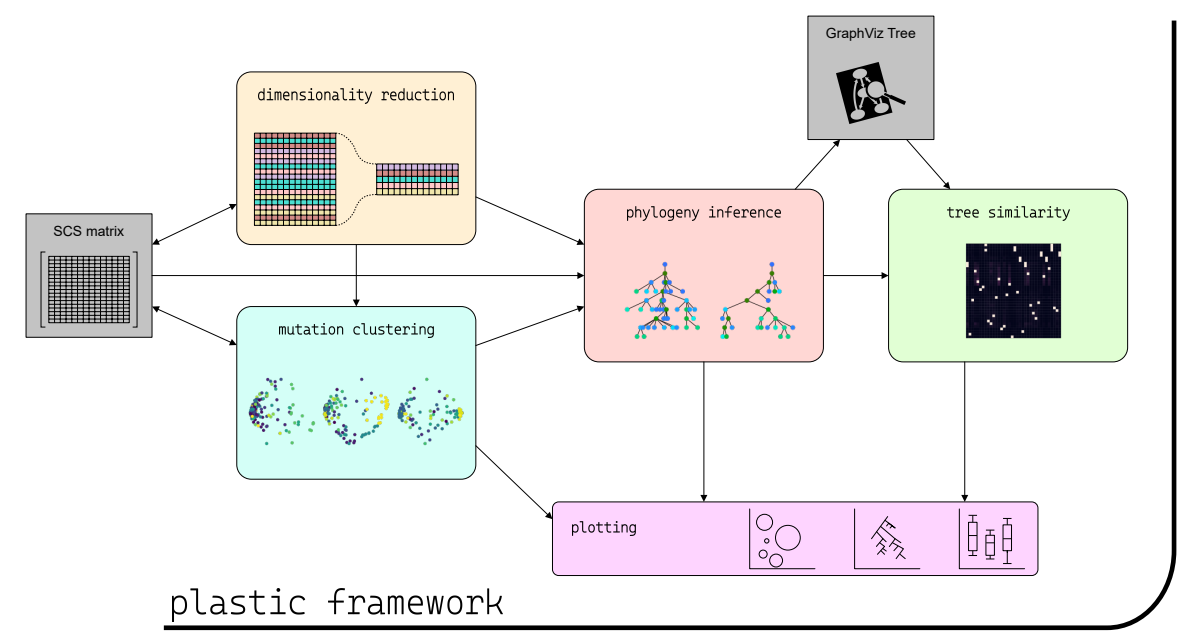

Figure 1: Graphical representation of the plastic framework and the interaction between its components and with the input/output files.

information across the methods.

In particular, plastic provides an SCS matrix data structure that is enriched with some additional information that can be shared among the different steps, and a phylogeny tree structure that is used for communication between the phylogeny inference and the phylogeny comparison steps. Such structures are transparent to the user and contribute many additional functionalities without complications from the end-user perspective.

Furthermore, given the nature of plastic, we added some graphical capabilities, so that each step of the pipeline can be displayed within an interactive notebook or be exported to separate files.

Finally, notice that each step is optional, therefore allowing the execution of the entire pipeline, or only of a part of it. A schematic of our plastic tool is depicted in Figure 1. We now introduce some of the new dimensionality reduction features we have added to plastic, as follows. 


\subsection{Dimensionality Reduction}

Dimensionality reduction is a popular approach to enhance the performance of machine learning algorithms and to avoid the problem of the "curse-ofdimensionality" Ali et al. (2019ab). To increase the clustering performance and reduce the runtime for clustering, we use two dimensionality reduction approaches, namely ridge regression Hoerl and Kennard (1970); Marquardt and Snee (1975) and autoencoder Wang et al. (2014).

The main goal of ridge regression $(\mathrm{RR})$ is to find a linear function, which models the dependencies between covariate variables and univariate labels. Although ridge regression is an older approach, it is still successfully used in or-

der to reduce the dimensions of current datasets Chen et al. (2018); Zhang et al. (2010). Ridge regression help to find a subspace, which most compactly expresses the target and rejects other possible but less compact candidates Imakura et al. (2019). It works by introducing a bias term - the goal of ridge regression is to increase the bias (by changing the slope of the regression line) in order to improve the variance (generalization capability). The general expression for ridge regression is as follows:

$$
\min \left(\text { sum of square residuals }+\alpha \times \operatorname{slope}^{2}\right)
$$

where $\left(\alpha \times\right.$ slope $\left.^{2}\right)$ is an $l_{2}$ penalty term. Ridge regression gives insights on which independent variables are not informative (independent variables for which we can reduce the slope close to zero). We can eliminate those independent variables to reduce the dimensions of the data. Note that after the dimensionality reduction, we are left with the variables from the actual data and not some latent variables.

Autoencoder (AE) is an unsupervised artificial neural network based approach used for dimensionality reduction Wang et al. (2012, 2016). It consists 
of three the components: encoder; code; and decoder (pictured in Figure 2). The encoder component compresses the input data and produces the code (low dimensional latent-space representation). The decoder component then reconstructs the input only using this low dimensional representation only (hence an unsupervised approach). The low-dimensional data (in the code component) is a compact summary, or compression of the input. This compact data is used as the reduced dimensional representation of the original data. The activation function used for the encoding component is the rectified linear activation function (ReLU), while we used the sigmoid function for the decoding. The loss function that we used in our experiments is the least squared error. The optimizer that we use is Adadelta Polic et al. (2019), a stochastic gradient descent method which is based on adaptive learning rate per dimension. It is a popular optimizer used in autoencoder because it avoids the continual decay of learning rates throughout training. It also helps to decide the global learning rate, hence not requiring to select it manually.

\section{Results}

Here we present our results on both real and simulated data.

\subsection{Real data}

As a result, we show a complete workflow example on a real medulloblastoma dataset Hovestadt et al. (2019). In particular, we will explore a full pipeline analysis where multiple samples are taken into account. For each sample: mutations are clustered using celluloid; phylogeny trees are reconstructed using $S A S C$; and finally, the patients are clustered using MP3treesim as similarity measure between them.

The sequencing study consists of 36 patients that we want to cluster into 


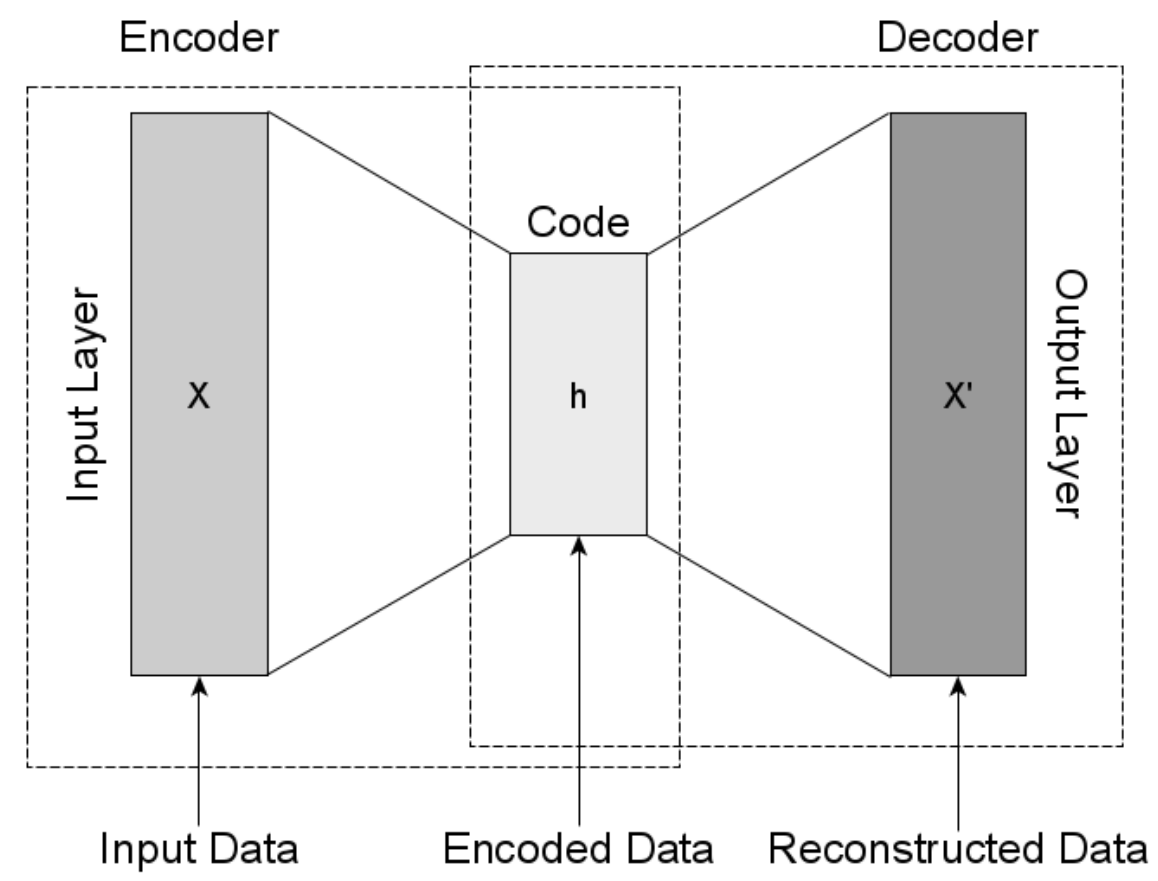

Figure 2: The autoencoder architecture. The encoder takes input data and maps it to a latent space (code component) of reduced dimension. The decoders code back the latent representation to the output. This architecture learns to compress data by reducing the reconstruction error (least squared error). 


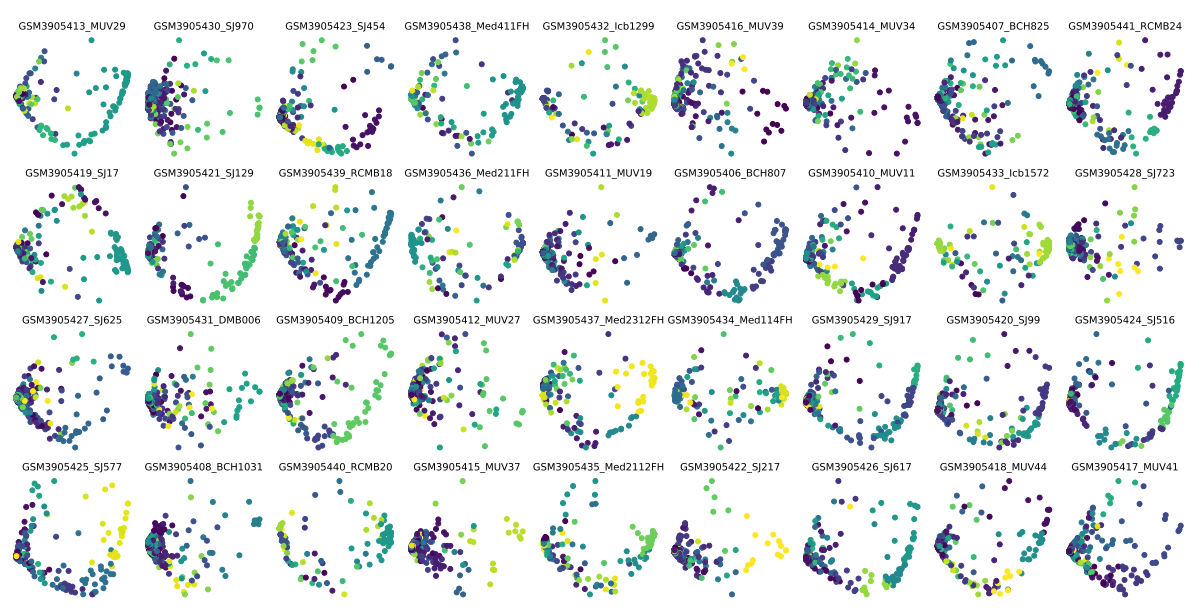

Figure 3: Mutations clustered on SCS data computed by the celluloid submodule and displayed by plastic for the 36 medulloblastoma patients in the dataset of Hovestadt et al. (2019).

different subtypes. To simulate a real case scenario, where such information is not available, we started from SCS binary matrices, one for each patient, and we clustered mutations using celluloid $(k=50)$, for which we can see a summary in Figure 3 .

After the first preprocessing step, we utilized the $S A S C$ submodule of plastic to infer the evolutionary trees of each patient. As a proof of concept we run it using the following parameters $\alpha=0.25, \beta=1 \times 10^{-4}, k=0, d=0$ for whose definitions we refer to $S A S C$ 's manuscript Ciccolella et al. (2020b). Once computed we used the newly-added plotting feature to display the phylogenies using the $S A S C$-viz plotting feature to prettify the trees; the result is shown in Figure 4

As the last step, we then used the MP3treesim submodule to compute a matrix of similarity-scores between all the trees and then used it to cluster the patients according to a hierarchical clustering method. We then displayed the final clustering of the patients in Figure 5 . 


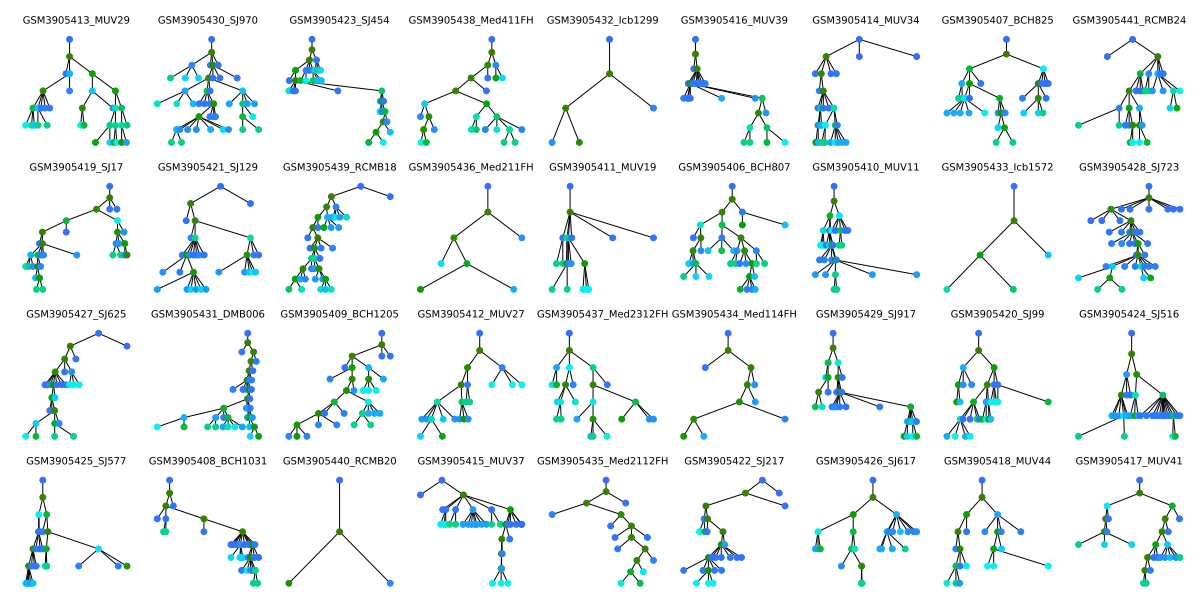

Figure 4: Trees computed by the $S A S C$ submodule and displayed by plastic for the 36 medulloblastoma patients in the dataset of Hovestadt et al. (2019).

\subsection{Simulated data}

To evaluate the performance of the input matrix reduction methods that we present here, we designed a two-fold experiment on synthetic datasets. We first measure the quality of the matrix obtained from the reduction step - in this case we have a ground truth that we can use for the evaluation. Second, we evaluate the accuracy and runtime of the downstream phylogeny inference tools when given as input the reduced instance from the first step, to assess that our reduction step is actually useful. This is similar to the experimental approach taken in Ciccolella et al. (2021).

The simulated data are generated as follows. First we generate a random tree topology on $s$ nodes, each representing a tumor clone, by first creating a root (the germline) and then iteratively attaching the $s-1$ remaining nodes uniformly at random to any other node in the tree. The nodes are then randomly labeled with $m$ mutations - each mutation understood as being acquired at the node that it labels. Then, a total of $n$ cells are randomly associated to the $s$ nodes. Each cell harbours all of the mutations on the path in the tree from the root to 


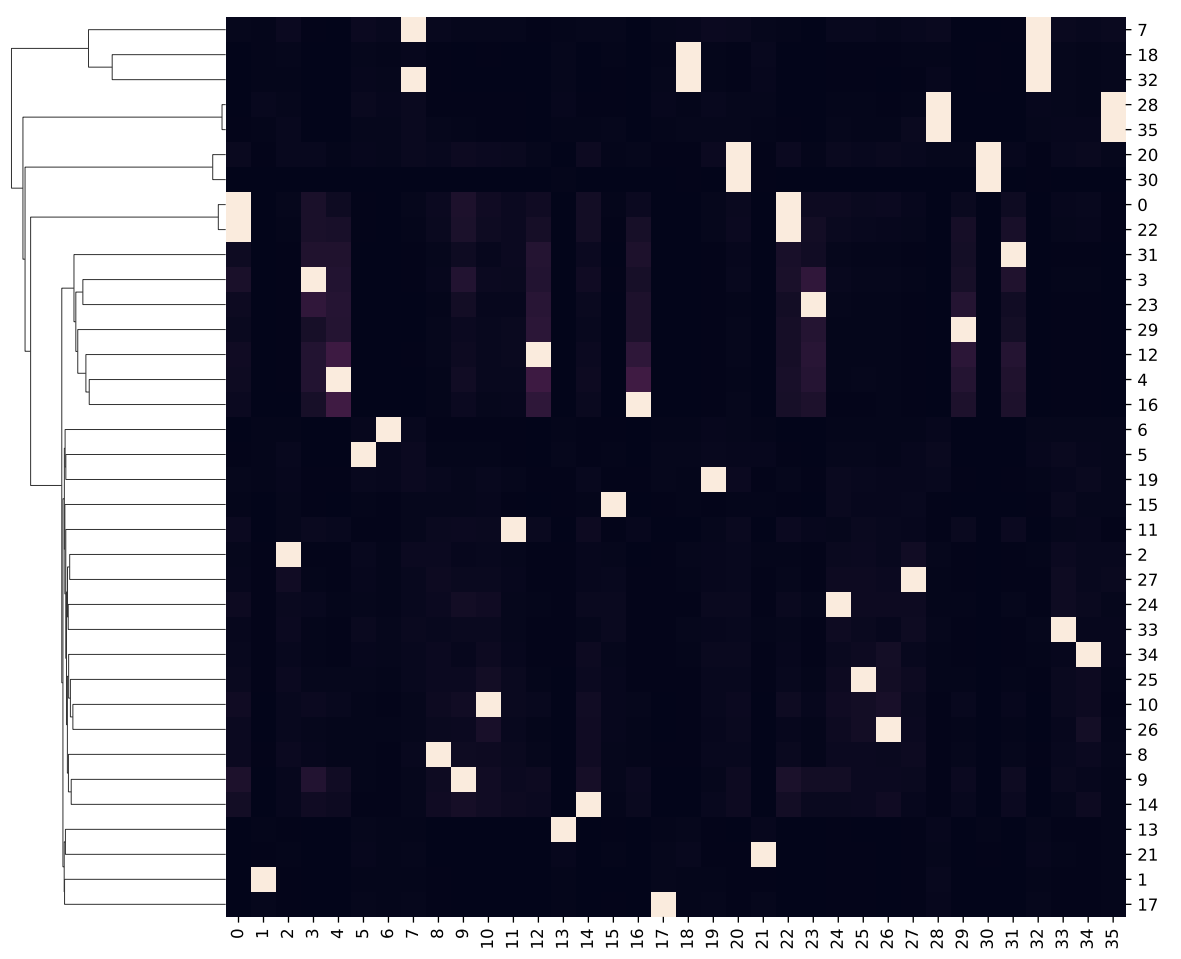

Figure 5: Clustermap based on the similarity between the trees computed by the mp3 submodule of plastic for the 36 medulloblastoma patients in the dataset of Hovestadt et al. (2019). 
the node that the cell labels. A binary $n \times m$ matrix $M$ is then obtained from the cells, where $M[i, j]=1$ if cell $i$ harbours mutation $j$, otherwise $M[i, j]=0$. Noise is then added to this matrix according to the false negative, false positive and missing value rates, to simulate a real single cell sequencing experiment. Each of the $s$ nodes is therefore considered as a natural (true) cluster of the simulated dataset.

We design three experiments, each with 100, 200 and 300 cells, respectively. In each experiment, we generate 50 simulated datasets with its corresponding number of cells, using the procedure mentioned above. In all three experiments, the number $s$ of clones is 20, and the number $m$ of mutations is 1000 .

To each dataset of our experiments, we first performed dimensionality reduction in the cells using both the ridge regression and autoencoder techniques mentioned in Section 2 The number of dimensions selected in case of autoencoder are 50, 100, and 150 for experiments 1,2 and 3, respectively. This number of dimensions, in each case, is selected empirically when the least squared error for the loss function is minimized. The loss function gives us the error value when autoencoder tries to reconstruct the input in the decoder component. The main goal in this case is to reconstruct the input as accurately as possible, giving rise to the respective numbers of dimensions above. The number of dimensions selected in the case of ridge regression is different for each dataset because ridge regression is a data driven technique, however the average number of dimensions for each of experiments 1, 2 and 3 is roughly 49, 97, and 146, respectively (see Table 1). Following dimensionality reduction in the cells, we then clustered the mutations using celluloid.

\subsubsection{Evaluating input matrix reduction.}

Because we are performing dimensionality reduction only on the cells, we can still use the same measures of precision and recall used in Ciccolella et al. (2021), 


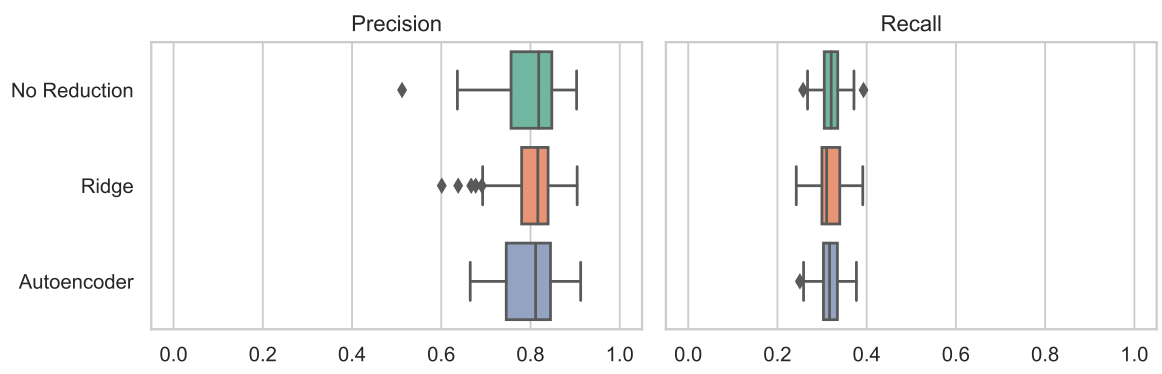

Figure 6: Precision and recall results of the clusterings of the mutations obtained for experiment 1, generated with 1000 mutations and 100 cells. The first row corresponds the results obtained by clustering the mutations to $k=100$ clusters using celluloid (no dimensionality reduction). The second and third rows correspond to first applying a dimensionality reduction step on the 100 cells, using ridge regression and autoencoder, respectively, followed by the clustering of the 1000 mutations to $k=100$ clusters (in the reduced number of cells) using celluloid.

non-branching path — however they cannot separate mutations that have been erroneously clustered together. It is for this same reason that the number $(k)$ of clusters is carefully chosen with high precision in mind.

On the other hand, ridge regression does not allow to determine a priori the number of cells that will be obtained. For this reason we report, in Table 1 , the distributions 1 of the actual number of cells obtained.

Figures 6, 7, and 8 correspond to the precision and recall of the clusterings of the mutations obtained after first applying dimensionality reduction in the cells of experiments 1,2 and 3 , respectively.

\subsubsection{Evaluating the effect of the reduction step on downstream phy- logeny inference}

The goal of dimensionality reduction (in the cells) and clustering (of the mutations) is to allow for a significant decrease in the runtime of the phylogeny inference, the most expensive step in the pipeline - as long as it does not worsen

\footnotetext{
${ }^{1}$ note that the variance of all three distributions is small
} 


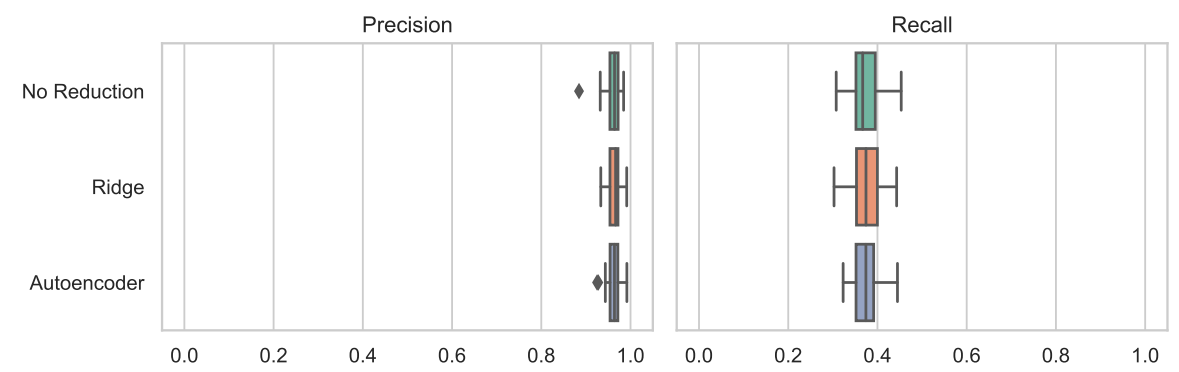

Figure 7: Precision and recall results of the clusterings of the mutations obtained for experiment 2, generated with 1000 mutations and 200 cells. The first row corresponds the results obtained by clustering the mutations to $k=100$ clusters using celluloid (no dimensionality reduction). The second and third rows correspond to first applying a dimensionality reduction step on the 200 cells, using ridge regression and autoencoder, respectively, followed by the clustering of the 1000 mutations to $k=100$ clusters (in the reduced number of cells) using celluloid.

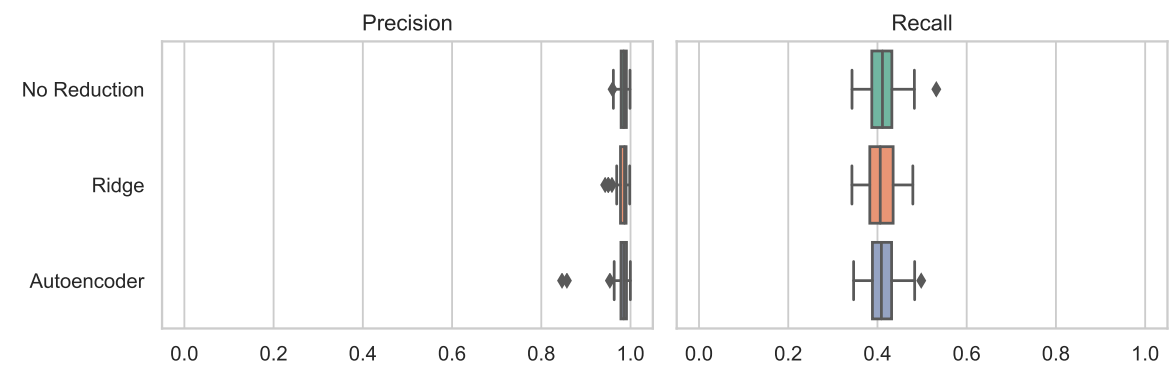

Figure 8: Precision and recall results of the clusterings of the mutations obtained for experiment 3, generated with 1000 mutations and 300 cells. The first row corresponds the results obtained by clustering the mutations to $k=100$ clusters using celluloid (no dimensionality reduction). The second and third rows correspond to first applying a dimensionality reduction step on the 300 cells, using ridge regression and autoencoder, respectively, followed by the clustering of the 1000 mutations to $k=100$ clusters (in the reduced number of cells) using celluloid. 
the accuracy. The idea is that, since the reduced matrix has much fewer rows and columns, using it in place of the original matrix as input to the phylogeny inference should result in much lower runtimes, at the risk of decreasing the accuracy of downstream analysis, due to errors introduced in the dimensionality reduction and clustering steps. The main observation is that, if the clusters are highly precise, the decrease of the accuracy of the downstream inference is negligible or small. Assessing this fact is the main goal of our experimental analysis, based on the measures used in Ciccolella et al. (2020c b):

Ancestor-descendant accuracy: For each pair $\left(m_{1}, m_{2}\right)$ of mutations such that $m_{1}$ is an ancestor of $m_{2}$ in the ground truth, we check whether $m_{1}$ is an ancestor of $m_{2}$ also in the inferred tree $(T P)$ or whether it is not $(F N)$. Moreover, each pair $\left(m_{1}, m_{2}\right)$ of mutations such that $m_{1}$ is an ancestor of $m_{2}$ in the inferred tree but not in the ground truth, we consider that pair a false positive.

Different lineages accuracy: Similarly to the previous measure, we check whether mutations in different branches (i.e., neither is an ancestor of the other) are correctly inferred or if any pair of mutation is erroneously inferred in different branches.

Since these new dimensionality reduction techniques are built into the plastic framework, one can customize a cancer phylogeny inference task by:

1. choosing any (or none of the) dimensionality reduction steps from ridge regression or autoencoder; followed by

2. clustering (or not) with celluloid; and finally

3. phylogeny inference with $S A S C$ in either the reduced set of cells, or not.

An extensive study of how clustering can reduce the runtime (and sometimes improve accuracy) of the downstream phylogeny inference appears in Ciccolella 
et al. (2021), and so here we explore the further gains in runtime and/or accuracy that might be achieved by adding a dimensionality reduction step. Hence, in our experiments, we try three different choices for the reduction step — ridge regression, autoencoder, and no dimensionality reduction to produce a matrix to be given to celluloid (notice that $S A S C$ will receive the matrix on the original set of cells, but with the clustered mutations). Moreover, when applying ridge regression, we also provide the reduced set of cells to $S A S C$, hence resulting in four different settings — when no dimensionality reduction is performed, the reduced and the original sets of cells are the same, while autoencoder produces reduced matrices in its learned latent tensor space, which is not an SCS matrix that can be fed to $S A S C$. We always perform the clustering step, because 1000 mutations is prohibitive for the downstream phylogeny inference (see Ciccolella et al. (2021)).

Figures 9, 10 and 11 report the ancestor-descendant and different lineages accuracy measures of the trees obtained by running plastic with the four settings mentioned above. From the results, it is clear that when dimensionality reduction is followed by inference in the original set of cells, there is no noticeable loss in accuracy. In these two cases, the clustering is still performed in the reduced set of cells, providing a speedup at no cost. On the other hand, we see a slight loss of accuracy in the ancestor-descendant measure when ridge regression is applied, and the resulting reduced set of cells is used also in the inference step.

We have investigated the speedup provided by ridge regression. In Figure 12 are represented the running times of celluloid with and without first applying ridge regression, as a function of the number of mutations. More precisely, we take the first columns of the datasets of Experiment 1 (the number of mutations is the $x$ axis). 


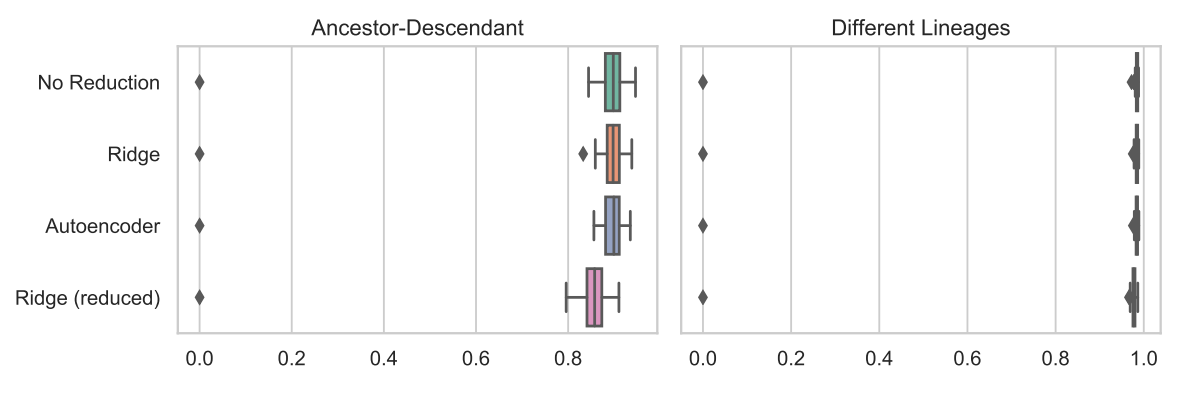

Figure 9: Ancestor-descendant and different lineages accuracies of the trees obtained by running plastic on the data of experiment 1 . From the top, the four rows correspond to the settings: no dimensionality reduction, just clustering - No Reduction —; ridge regression + clustering in the original set of cells Ridge - ; autoencoder + clustering in the original set - Autoencoder —; and ridge regression + clustering in the reduced set - Ridge(reduced).

Table 2: Runtimes of $S A S C$ on the experiments $^{a}$

\begin{tabular}{lll}
\hline Experiment & celluloid & RR + celluloid \\
\hline 1 & $37.7 \pm 24.3$ & $0.414 \pm 0.0710$ \\
2 & $44.3 \pm 21.2$ & $0.832 \pm 0.0682$ \\
3 & $34.2 \pm 25.6$ & $1.31 \pm 0.112$ \\
\hline
\end{tabular}

${ }^{a}$ The mean $(\mu) \pm$ standard deviation $(\sigma)$ of the runtime of $S A S C$ on the 50 datasets of each of the three experiments (on the original set of cells), after having either: clustered the mutations of the input matrix using celluloid (celluloid); or performed dimensionality reduction on the cells using ridge regression, followed by clustering the mutations with celluloid (RR + celluloid). Times are reported in hours, and to three significant digits.

Finally, in Table 2 we report the running times of $S A S C$ when ridge regression is applied, and the resulting reduced set of cells is used also in the inference step, that is, the case where we have observed a slight loss of accuracy in the ancestor-descendant measure. In this case the phylogeny inference is up to 100 times faster, making therefore possible to run $S A S C$ on more mutations. 


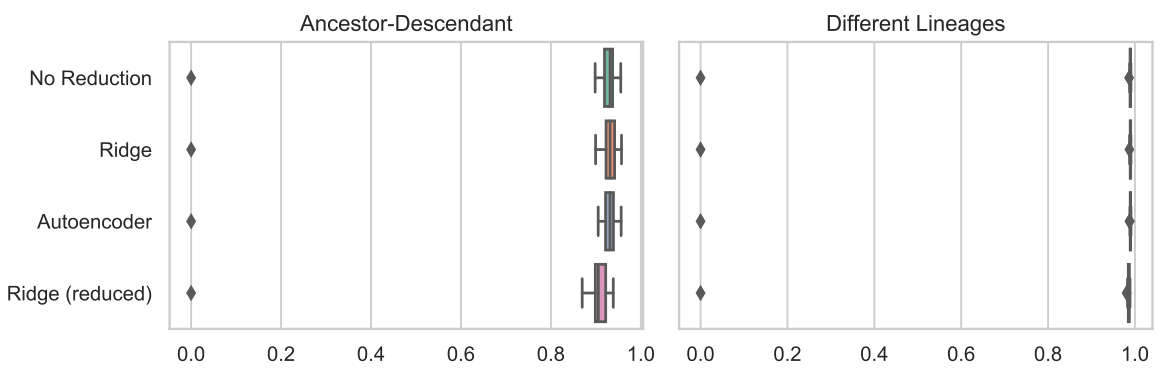

Figure 10: Ancestor-descendant and different lineages accuracies of the trees obtained by running plastic on the data of experiment 2. From the top, the four rows correspond to the settings: no dimensionality reduction, just clustering - No Reduction - ; ridge regression + clustering in the original set of cells Ridge -; autoencoder + clustering in the original set - Autoencoder - ; and ridge regression + clustering in the reduced set - Ridge(reduced).

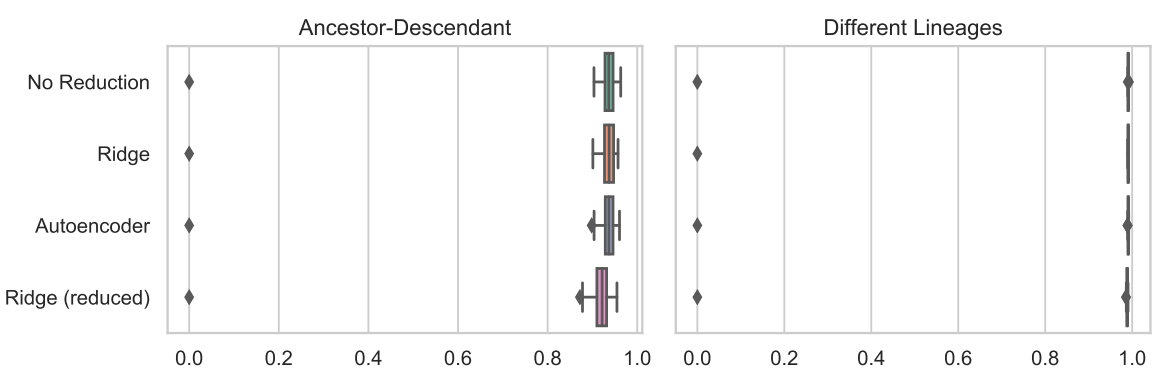

Figure 11: Ancestor-descendant and different lineages accuracies of the trees obtained by running plastic on the data of experiment 3. From the top, the four rows correspond to the settings: no dimensionality reduction, just clustering - No Reduction - ; ridge regression + clustering in the original set of cells Ridge -; autoencoder + clustering in the original set - Autoencoder —; and ridge regression + clustering in the reduced set - Ridge(reduced). 


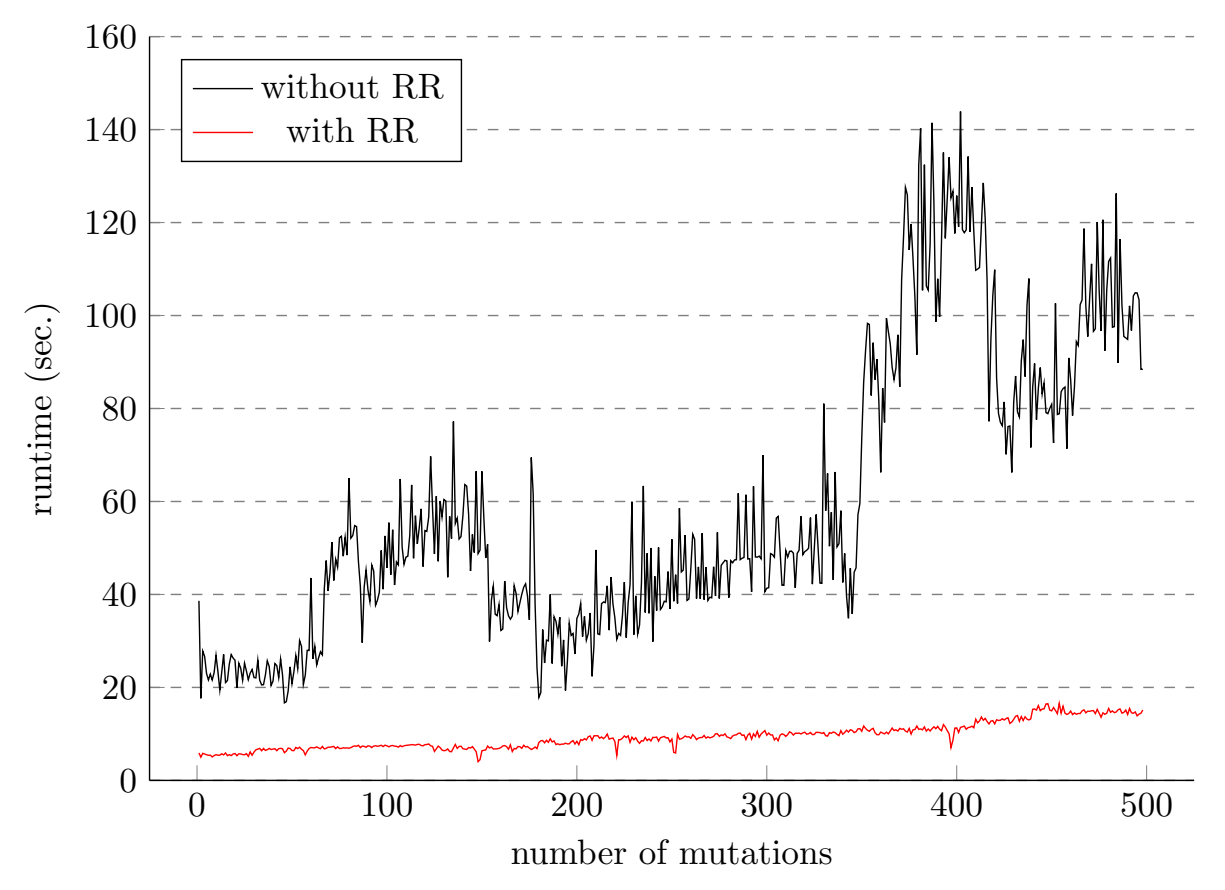

Figure 12: Runtime analysis, as a function of the number of mutations, of the clustering step (celluloid) without dimensionality reduction using ridge regression (in black), and with ridge regression (in red). 


\section{Discussion}

Given the large amount of SCS cancer studies that are being published, there is a need for a fast and easy-to-use framework that allows to perform the needed analyses. We developed plastic with this goal in mind, a pipeline composed of different submodules that allow for dimensionality reduction on SCS cells, clustering of SCS mutations, inference of tumor phylogenies, comparison of such trees, and convenient plotting of the results.

Each of the submodules can be used independently or they can be used in conjunction with each other to create complex operations, due to special data structures developed for the interaction between different methods.

The plastic pipeline is open-source and available at https://github.com/ plastic-phy along with extensive documentation and a Jupyter Notebook that replicates the real-case scenario depicted in Section 3.1 .

Future improvements for plastic would be to include more tools into the pipeline to extend the breadth of analyses available and to provide additional algorithmic alternatives for the same task. Moreover, we could incorporate more steps, such as the removal of doublets, to streamline the entire pipeline even more.

\section{Acknowledgments}

We thank Paola Bonizzoni and Iman Hajirasouliha for many useful discussions on the topic.

\section{References}

Abeßer, J., Mimilakis, S.I., Gräfe, R., et al. 2017. Acoustic scene classification by combining autoencoder-based dimensionality reduction and convolu- 
tional neural networks. In Proc. of the Detection and Classification of Acoustic Scenes and Events 2017 Workshop (DCASE2017). pages 7-11.

Ali, S., Mansoor, H., Arshad, N., et al. 2019a. Short term load forecasting using smart meter data. In Proceedings of the Tenth ACM International Conference on Future Energy Systems. pages 419-421.

Ali, S., Mansoor, H., Khan, I., et al. 2019b. Short-term load forecasting using ami data. arXiv preprint arXiv:1912.12479 .

Anderberg, M. 1973. Cluster Analysis for Applications. Academic Press.

Bernardini, G., Bonizzoni, P., Della Vedova, G., et al. 2019. A rearrangement distance for fully-labelled trees. In 30th Annual Symposium on Combinatorial Pattern Matching (CPM 2019). Schloss Dagstuhl-Leibniz-Zentrum fuer Informatik.

Bernardini, G., Bonizzoni, P., and Gawrychowski, P. 2020. On Two Measures of Distance Between Fully-Labelled Trees. In I.L. Gørtz and O. Weimann, editors, 31st Annual Symposium on Combinatorial Pattern Matching (CPM 2020). volume 161 of Leibniz International Proceedings in Informatics (LIPIcs). Schloss Dagstuhl-Leibniz-Zentrum für Informatik. pages 6:1-6:16.

Bonizzoni, P., Braghin, C., Dondi, R., et al. 2012. The binary perfect phylogeny with persistent characters. Theoretical Computer Science 454, 51-63.

Bonizzoni, P., Carrieri, A.P., Della Vedova, G., et al. 2017. A colored graph approach to perfect phylogeny with persistent characters. Theoretical Computer Science 658, 60-73.

Bonizzoni, P., Ciccolella, S., Della Vedova, G., et al. 2018. Does relaxing the infinite sites assumption give better tumor phylogenies? an ILP-based com- 
parative approach. IEEE/ACM Transactions on Computational Biology and Bioinformatics , 1-1.

Brown, D., Smeets, D., Székely, B., et al. 2017. Phylogenetic analysis of metastatic progression in breast cancer using somatic mutations and copy number aberrations. Nature Communications 8, 14944 EP -.

Chen, S.B., Ding, C.H., and Luo, B. 2018. Linear regression based projections for dimensionality reduction. Information Sciences 467, 74-86.

Ciccolella, S., Bernardini, G., Denti, L., et al. 2020a. Triplet-based similarity score for fully multi-labeled trees with poly-occurring labels. Bioinformatics

Ciccolella, S., Patterson, M., Bonizzoni, P., et al. 2021. Effective clustering for single cell sequencing cancer data. IEEE Journal of Biomedical and Health Informatics .

Ciccolella, S., Ricketts, C., Soto Gomez, M., et al. 2020b. Inferring cancer progression from Single-Cell Sequencing while allowing mutation losses. Bioinformatics $37,3,326-333$.

Ciccolella, S., Soto Gomez, M., Patterson, M.D., et al. 2020c. Gpps: An ILPbased approach for inferring cancer progression with mutation losses from single cell data. BMC Bioinformatics 21, 1, 413.

D’Agostino, D., Serani, A., Campana, E.F., et al. 2018. Deep autoencoder for off-line design-space dimensionality reduction in shape optimization. In 2018 AIAA/ASCE/AHS/ASC Structures, Structural Dynamics, and Materials Conference. page 1648.

DePasquale, E.A., Schnell, D.J., Camp, P.J.V., et al. 2019. Doubletdecon: De- 
convoluting doublets from single-cell rna-sequencing data. Cell Reports 29, 6, $1718-1727 . e 8$.

DiNardo, Z., Tomlinson, K., Ritz, A., et al. 2019. Distance measures for tumor evolutionary trees. Bioinformatics Btz869.

El-Kebir, M. 2018. Sphyr: tumor phylogeny estimation from single-cell sequencing data under loss and error. Bioinformatics 34, 17, i671-i679.

El-Kebir, M., Satas, G., Oesper, L., et al. 2016. Inferring the mutational history of a tumor using multi-state perfect phylogeny mixtures. Cell Systems 3, 1, $43-53$.

Farris, J.S. 1977. Phylogenetic analysis under dollo's law. Systematic Biology $26,1,77-88$.

Govek, K., Sikes, C., and Oesper, L. 2018. A consensus approach to infer tumor evolutionary histories. In Proceedings of the 2018 ACM International Conference on Bioinformatics, Computational Biology, and Health Informatics. pages $63-72$.

Gusfield, D. 1991. Efficient algorithms for inferring evolutionary trees. Networks $21,19-28$.

Hajirasouliha, I., Mahmoody, A., and Raphael, B.J. 2014. A combinatorial approach for analyzing intra-tumor heterogeneity from high-throughput sequencing data. Bioinformatics 30, 12, i78-i86.

Hoerl, A.E. and Kennard, R.W. 1970. Ridge regression: Biased estimation for nonorthogonal problems. Technometrics 12, 1, 55-67.

Hovestadt, V., Smith, K.S., Bihannic, L., et al. 2019. Resolving medulloblastoma cellular architecture by single-cell genomics. Nature 572, 7767, 74-79. 
Hu, Q. and Greene, C.S. 2018. Parameter tuning is a key part of dimensionality reduction via deep variational autoencoders for single cell rna transcriptomics. In BIOCOMPUTING 2019: Proceedings of the Pacific Symposium. World Scientific. pages $362-373$.

Imakura, A., Matsuda, M., Ye, X., et al. 2019. Complex moment-based supervised eigenmap for dimensionality reduction. In Proceedings of the $A A A I$ Conference on Artificial Intelligence. volume 33. pages 3910-3918.

Jahn, K., Beerenwinkel, N., and Zhang, L. 2021. The Bourque distances for mutation trees of cancers. Algorithms for Molecular Biology 16, 1, 9.

Jahn, K., Kuipers, J., and Beerenwinkel, N. 2016. Tree inference for single-cell data. Genome Biology 17, 1, 86.

Jiao, W., Vembu, S., Deshwar, A.G., et al. 2014. Inferring clonal evolution of tumors from single nucleotide somatic mutations. BMC Bioinformatics 15, 1, 35.

Karpov, N., Malikic, S., Rahman, M.K., et al. 2019. A multi-labeled tree dissimilarity measure for comparing "clonal trees" of tumor progression. Algorithms for Molecular Biology 14, 1, 17.

Kharchenko, P.V. 2021. The triumphs and limitations of computational methods for scRNA-seq. Nature Methods , 1-10.

Kimura, M. 1969. The number of heterozygous nucleotide sites maintained in a finite population due to steady flux of mutations. Genetics 61, 4, 893-903.

Kuipers, J., Jahn, K., Raphael, B.J., et al. 2017. Single-cell sequencing data reveal widespread recurrence and loss of mutational hits in the life histories of tumors. Genome Research . 
Liu, Y., Zhang, R., Nie, F., et al. 2019. Supervised dimensionality reduction methods via recursive regression. IEEE transactions on neural networks and learning systems 31, 9, 3269-3279.

Malikic, S., Jahn, K., Kuipers, J., et al. 2017. Integrative inference of subclonal tumour evolution from single-cell and bulk sequencing data. bioRxiv .

Malikic, S., McPherson, A.W., Donmez, N., et al. 2015. Clonality inference in multiple tumor samples using phylogeny. Bioinformatics 31, 9, 1349-1356.

Marass, F., Mouliere, F., Yuan, K., et al. 2016. A phylogenetic latent feature model for clonal deconvolution. Ann. Appl. Stat. 10, 4, 2377-2404.

Marquardt, D.W. and Snee, R.D. 1975. Ridge regression in practice. The American Statistician 29, 1, 3-20.

McQueen, J. 1967. Some methods for classification and analysis of multivariate observations. In the 5th Berkely Symposium on Mathematical Statistics and Probability. pages 281-297.

Polic, M., Krajacic, I., Lepora, N., et al. 2019. Convolutional autoencoder for feature extraction in tactile sensing. IEEE Robotics and Automation Letters 4, 4, 3671-3678.

Popic, V., Salari, R., Hajirasouliha, I., et al. 2015. Fast and scalable inference of multi-sample cancer lineages. Genome Biol 16, 1, 91.

Ramamurthy, M., Robinson, Y.H., Vimal, S., et al. 2020. Auto encoder based dimensionality reduction and classification using convolutional neural networks for hyperspectral images. Microprocessors and Microsystems 79, 103280.

Ramazzotti, D., Graudenzi, A., De Sano, L., et al. 2019. Learning mutational graphs of individual tumour evolution from single-cell and multi-region sequencing data. BMC bioinformatics 20, 1, 1-13. 
Rogozin, I., Wolf, Y., Babenko, V., et al. 2006. Dollo parsimony and the reconstruction of genome evolution. Oxford University Press.

Ross, E.M. and Markowetz, F. 2016. Onconem: inferring tumor evolution from single-cell sequencing data. Genome Biology 17, 1, 69.

Salehi, S., Steif, A., Roth, A., et al. 2017. ddclone: joint statistical inference of clonal populations from single cell and bulk tumour sequencing data. Genome Biology 18, 1, 44.

Satas, G. and Raphael, B.J. 2017. Tumor phylogeny inference using treeconstrained importance sampling. Bioinformatics 33, 14, i152-i160.

Singer, J., Kuipers, J., Jahn, K., et al. 2018. Single-cell mutation identification via phylogenetic inference. Nature Communications 9, 1, 5144.

Strino, F., Parisi, F., Micsinai, M., et al. 2013. Trap: a tree approach for fingerprinting subclonal tumor composition. Nucleic Acids Res 41, 17, e165-e165.

Toosi, H., Moeini, A., and Hajirasouliha, I. 2019. BAMSE: Bayesian model selection for tumor phylogeny inference among multiple samples. BMC Bioinformatics 20, 11, 282.

Wang, J., He, H., and Prokhorov, D.V. 2012. A folded neural network autoencoder for dimensionality reduction. Procedia Computer Science 13, 120-127.

Wang, W., Huang, Y., Wang, Y., et al. 2014. Generalized autoencoder: A neural network framework for dimensionality reduction. In Proceedings of the IEEE conference on computer vision and pattern recognition workshops. pages 490497.

Wang, Y., Yao, H., and Zhao, S. 2016. Auto-encoder based dimensionality reduction. Neurocomputing 184, 232-242. 
Wu, Y. 2019. Accurate and efficient cell lineage tree inference from noisy single cell data: the maximum likelihood perfect phylogeny approach. Bioinformatics $36,3,742-750$.

Yuan, K., Sakoparnig, T., Markowetz, F., et al. 2015. Bitphylogeny: a probabilistic framework for reconstructing intra-tumor phylogenies. Genome Biology $16,1,36$.

Zafar, H., Navin, N., Chen, K., et al. 2019. Siclonefit: Bayesian inference of population structure, genotype, and phylogeny of tumor clones from singlecell genome sequencing data. Genome Research .

Zafar, H., Tzen, A., Navin, N., et al. 2017. Sifit: inferring tumor trees from single-cell sequencing data under finite-sites models. Genome Biology 18, 1, 178.

Zhang, Z., Dai, G., Xu, C., et al. 2010. Regularized discriminant analysis, ridge regression and beyond. The Journal of Machine Learning Research 11, 2199 2228. 